Supporting information

\title{
Superhydrophobic candle soot as a low fouling stable coating on water treatment membrane feed spacers
}

Chidambaram Thamaraiselvan ${ }^{1,2}$, Emily Manderfeld ${ }^{3}$, Maurício Nunes Kleinberg ${ }^{4,2}$, Axel Rosenhahn $^{3 *}$ and Christopher J. Arnusch ${ }^{2 *}$

${ }^{1}$ The Jacob Blaustein Center for Scientific Cooperation, The Jacob Blaustein Institutes for Desert Research, Ben-Gurion University of the Negev, Sede-Boqer Campus, Midreshet Ben Gurion, 8499000, Israel

${ }^{2}$ Department of Desalination and Water Treatment, Zuckerberg Institute for Water Research, The Jacob Blaustein Institutes for Desert Research, Ben-Gurion University of the Negev, Sede-Boqer Campus, Midreshet Ben Gurion, 8499000, Israel

${ }^{3}$ Analytical Chemistry - Biointerfaces, Faculty for Chemistry and Biochemistry, Ruhr University Bochum, 44780 Bochum, Germany

${ }^{4}$ Albert Katz International School for Desert Studies, The Jacob Blaustein Institutes for Desert Research, Ben-Gurion University of the Negev, Sede-Boqer Campus, Midreshet Ben Gurion, 8499000, Israel

*Corresponding authors: C. J. Arnusch, arnusch@,bgu.ac.il; A. Rosenhahn, axel.rosenhahn@rub.de 


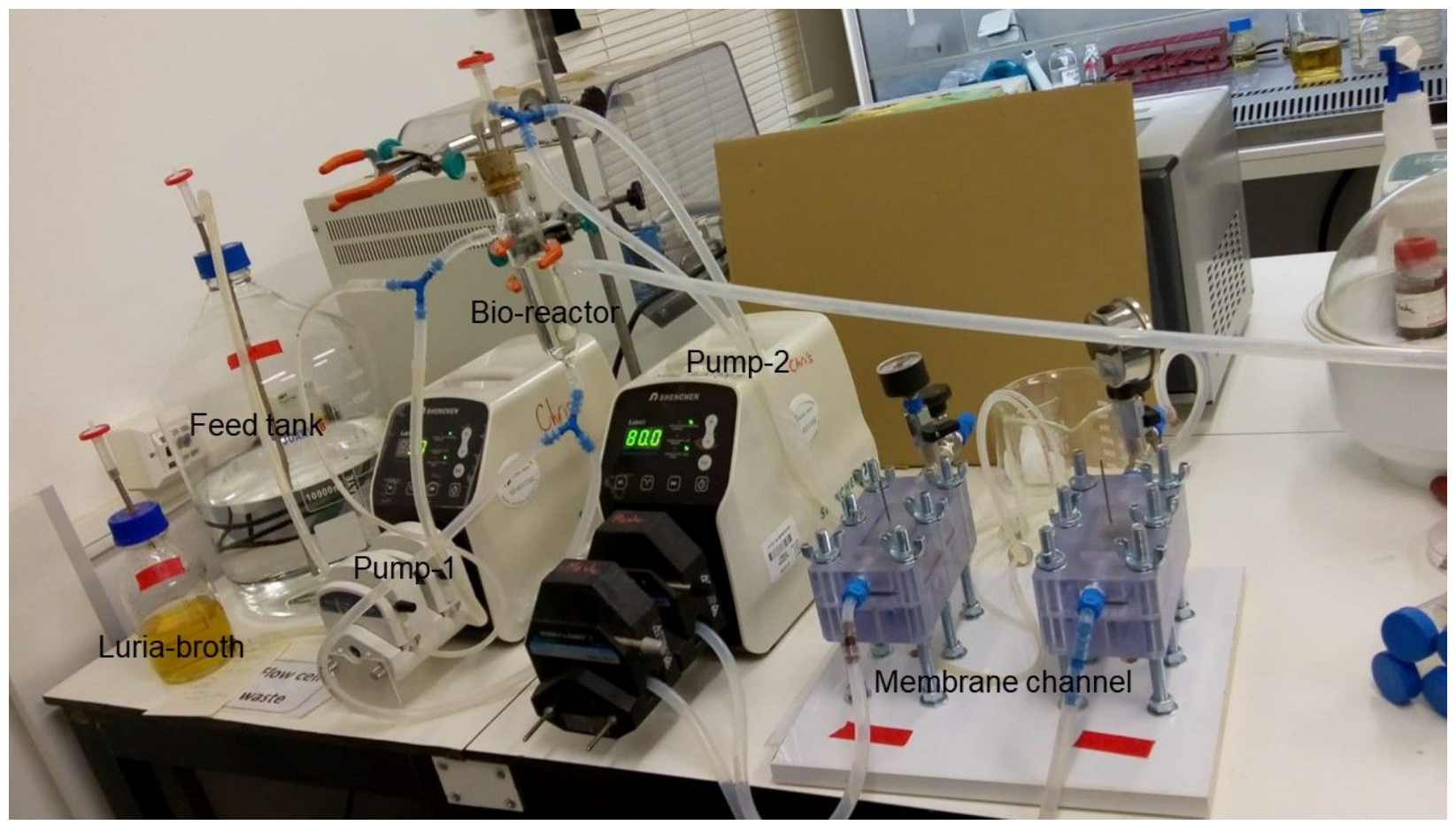

Figure S1: Apparatus for the fouling tests performed with the candle soot nanoparticles (CSNPs) modified spacer. 


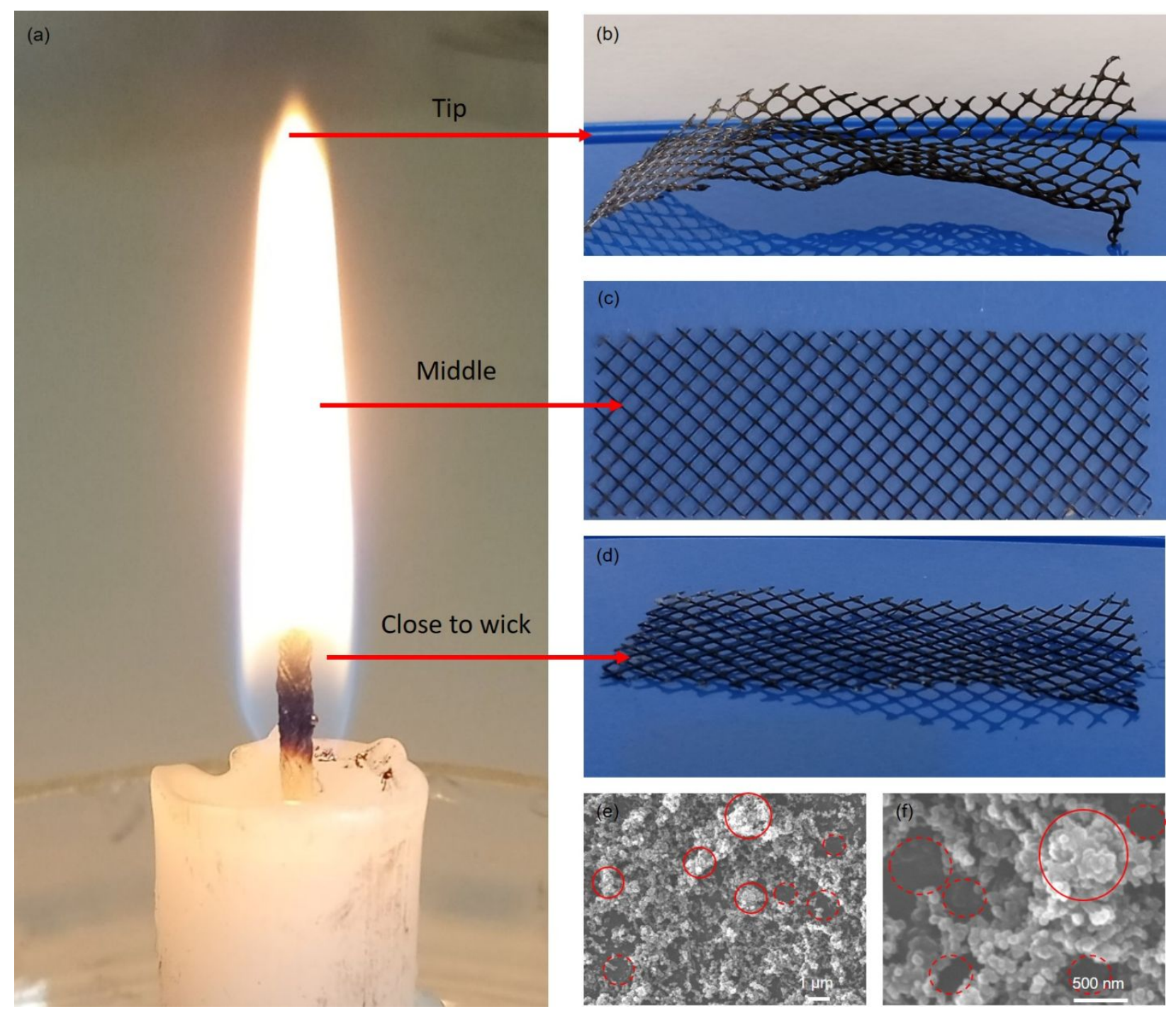

Figure S2. a) optical image of the candle flame b) CSNPs deposited spacer at the tip of the candle flame c) CSNPs deposited spacer at the middle of the flame d) CSNPs deposited spacer at the bottom (close to the wick) of the flame, e,f) SEM micrographs of the image (d) at different magnification (red circles show the agglomeration and dotted circle shows the non-uniform particle deposition. 
a)

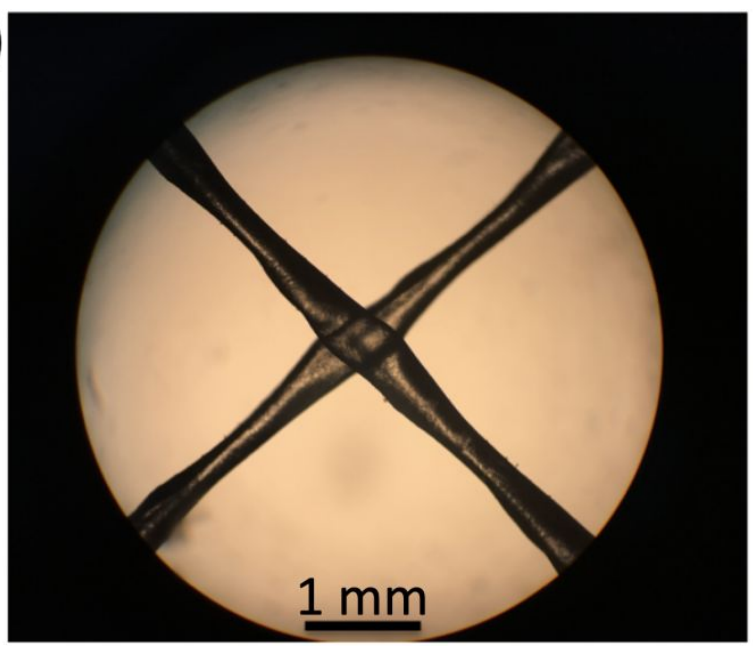

b)

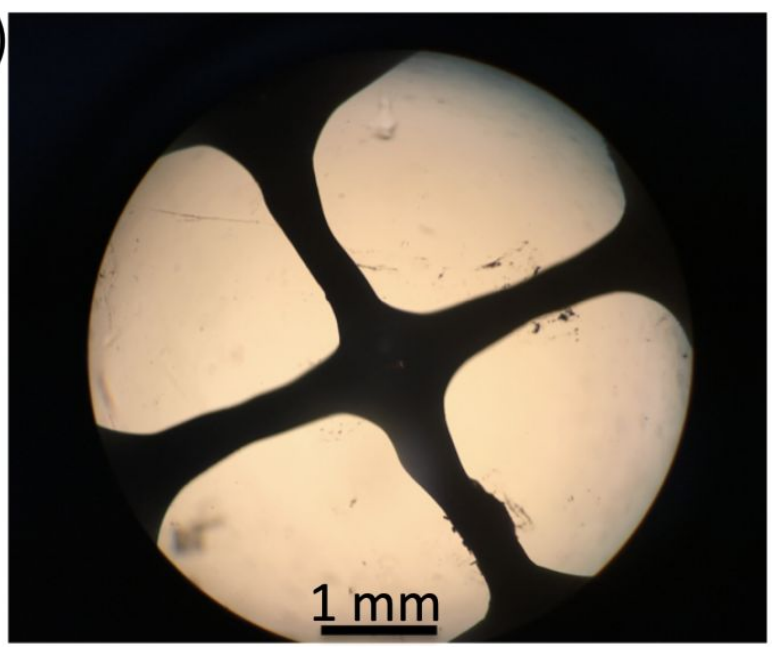

Figure S3. Images of the a) uncoated spacers and b) CSNPs-coated spacer observed by light microscope. The thickness of the fibers were measured using ImageJ. The average of 11 measurements was reported. Uncoated fiber thickness: $326 \pm 53 \mu \mathrm{m}$, CSNPs-coated fiber thickness: $418 \pm 66 \mu \mathrm{m}$.

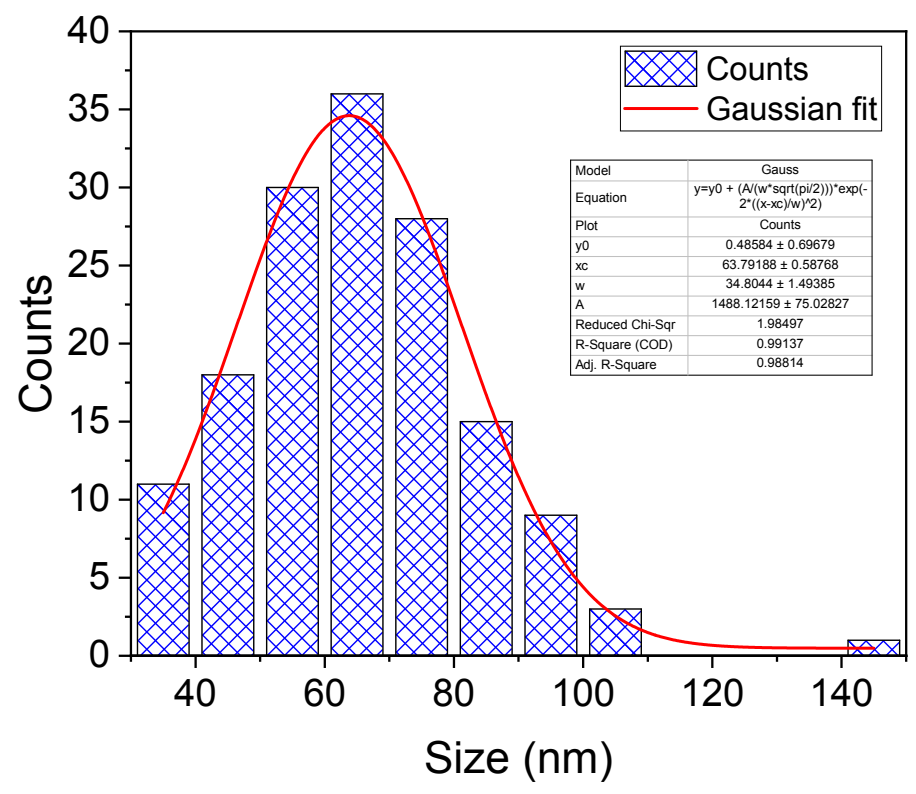

Figure S4. The size distribution of 150 counted CSNPs measured and calculated using Image J software. 

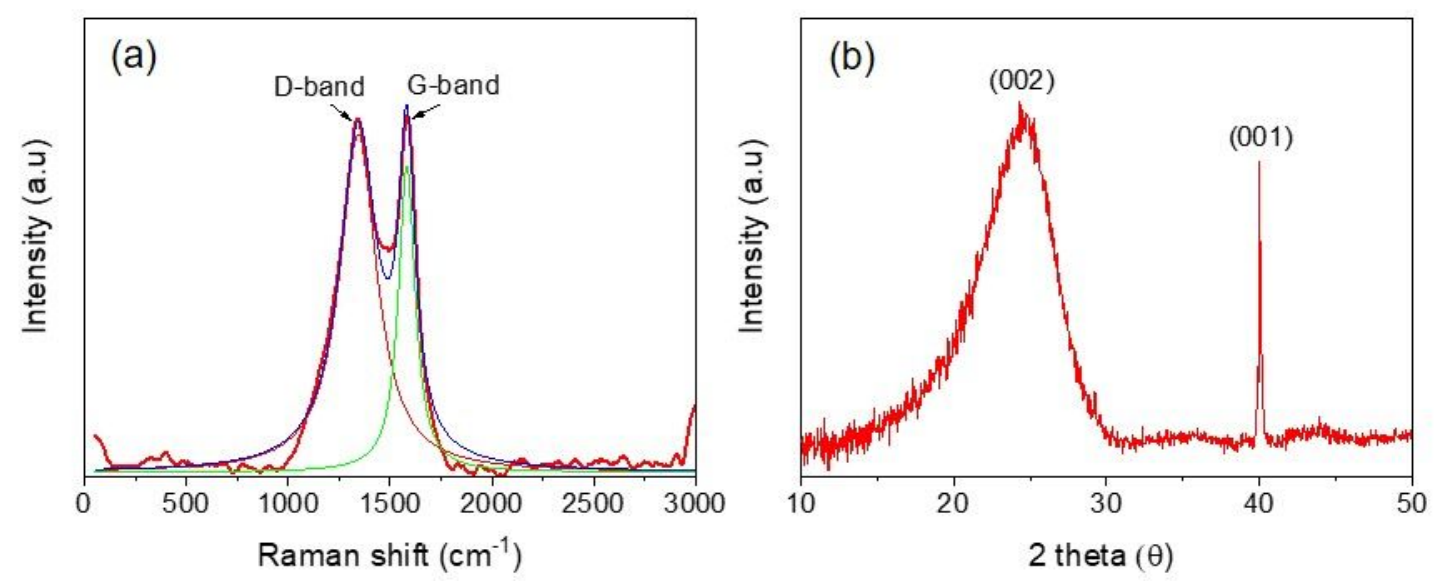

Figure S5. a) The deconvolution of the Raman spectrum of the CSNPs surface, fitted with Lorentz model. The Id/Ig value is 0.85 . b) X-ray powder diffraction of CSNPs.

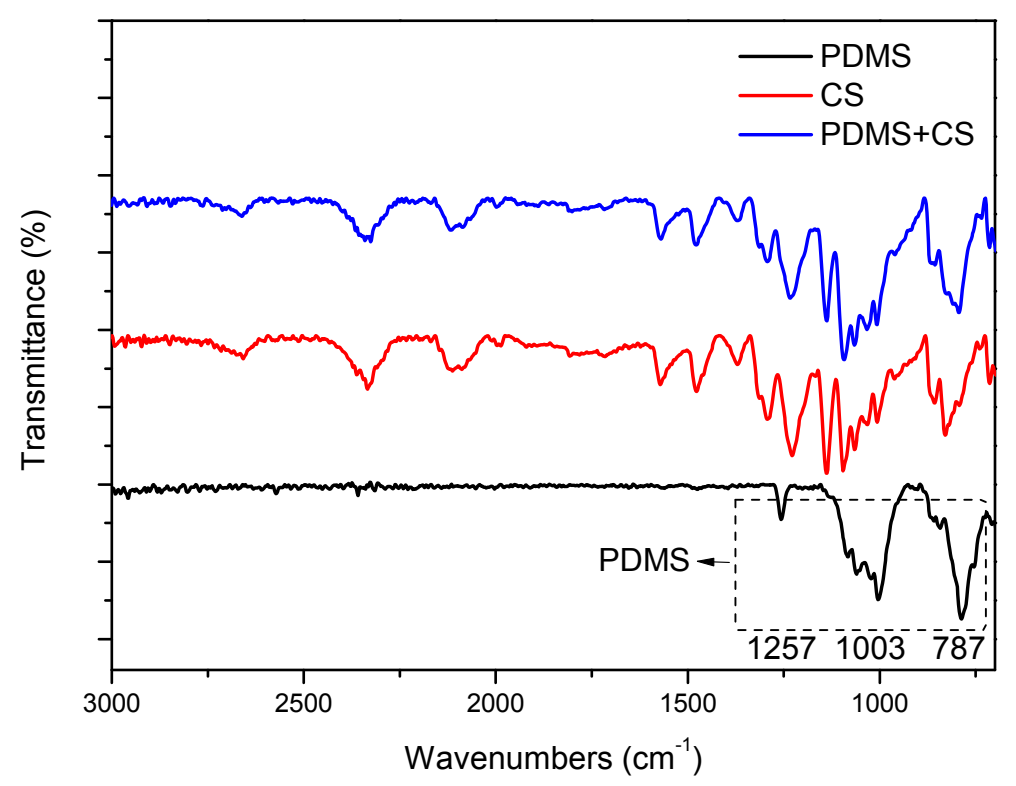

Figure S6. FTIR spectra of the samples PDMS (black line), CSNPs (red line), and PDMS+CSNPs (blue line). 

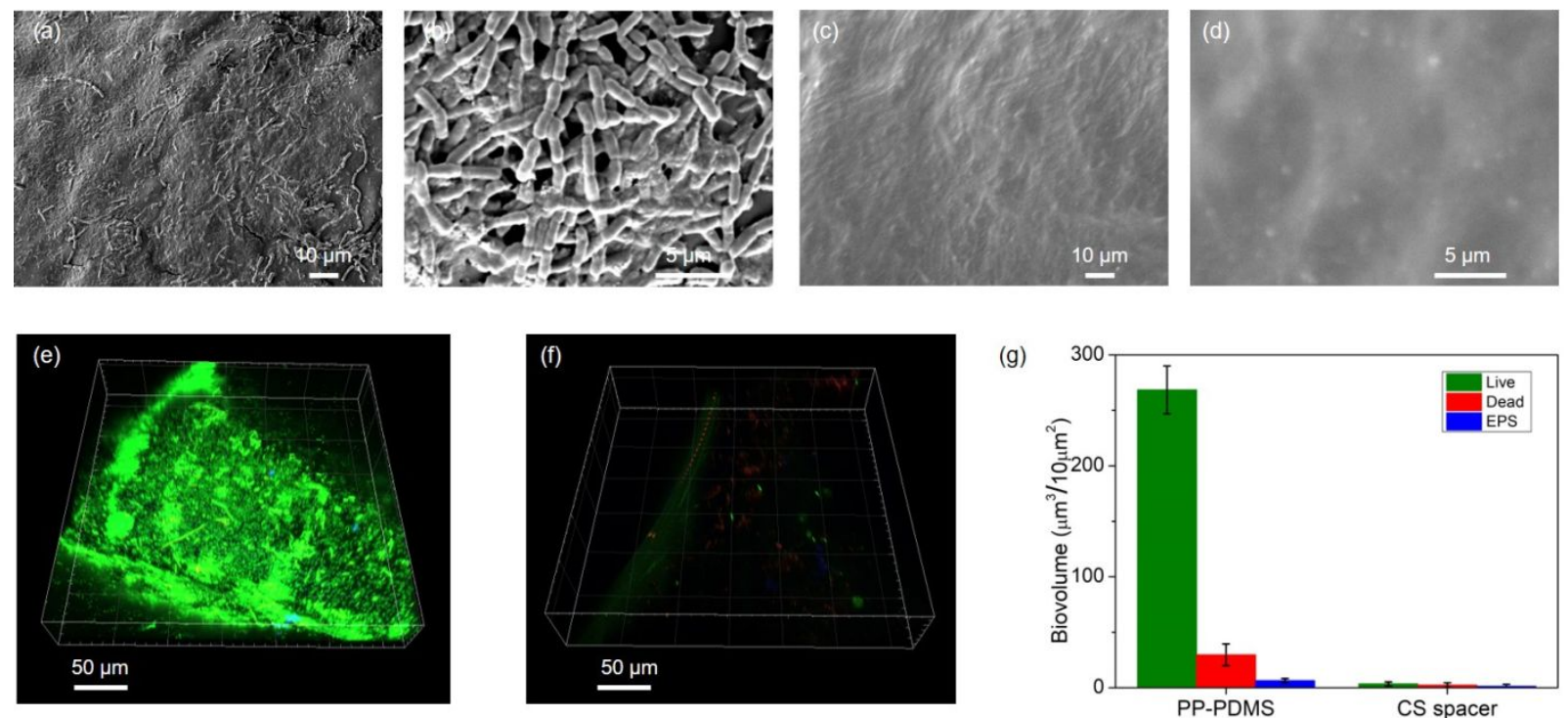

Figure S7. Biofouling analysis on the spacer surfaces using real brackish water as a feed water. Biofilm growth conditions include $P$. aeruginosa inoculation and incubation at $30{ }^{\circ} \mathrm{C}$ for $72 \mathrm{~h}$ under flow through mode. After fixation, SEM images of a-b) control and c-d) candle soot spacer were seen. CLSM images for e) control (PDMS coated PP spacer), and f) CSNPs spacer. g) Imaris quantification of the biofilm volume shows the live (green) dead (red) and EPS (blue). 


\section{Antibiofouling test in batch mode}

The control and modified spacers were tested for microbial fouling in non-flow conditions (batch mode) as follows. Sterile LB $(50 \mathrm{~mL})$ and a test sample spacer $2 \mathrm{~cm} \times 2 \mathrm{~cm}$ was added to a sterile $100 \mathrm{~mL}$ Erlenmeyer flask. A suspension of P.aeruginosa (PA01) was added for a final concentration of $10^{3} \mathrm{CFU} \mathrm{mL}-1$. The flasks were covered and were incubated in a rotary shaker (100 rpm) for up to $48 \mathrm{~h}$ at $30^{\circ} \mathrm{C}$. Afterwards, the spacers were taken, thoroughly washed with a sterile saline solution, and analyzed by SEM. Images were taken from at least 2 to 3 random areas on the surface and the images presented below are representative of the average of the entire surface (Figure S8). All components of the setup were then cleaned with a $70 \%$ ethanol solution and distilled water.
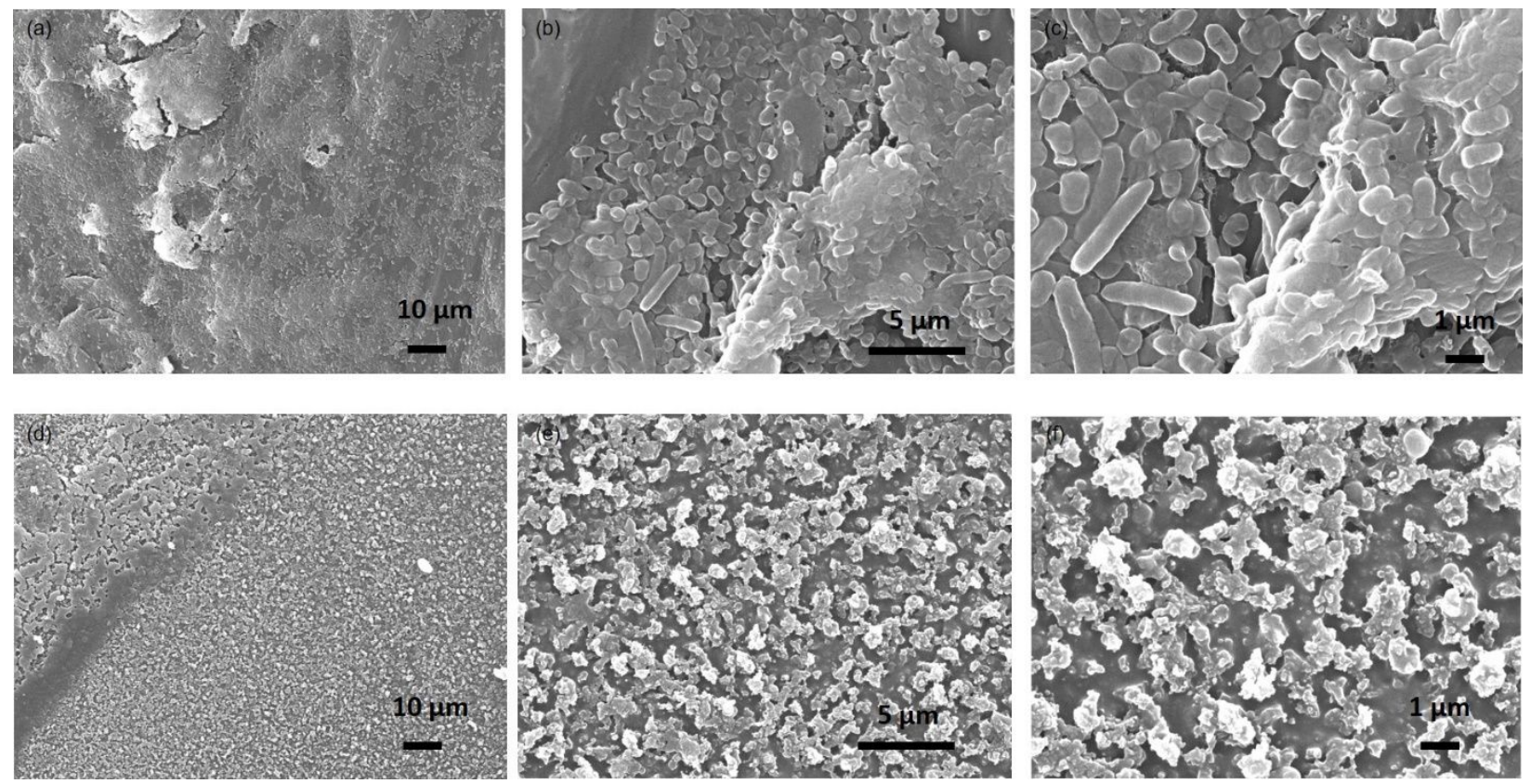

Figure S8. SEM micrographs of the feed spacer tested for biofouling activity at $30{ }^{\circ} \mathrm{C}$ for $48 \mathrm{~h}$ under batch mode growing bacteria conditions. Control spacer with different magnification $(a, b, c)$. Candle soot spacer with different magnification (d,e,f). 
(a)

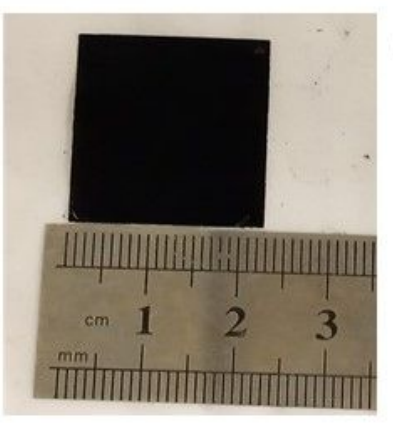

(b)

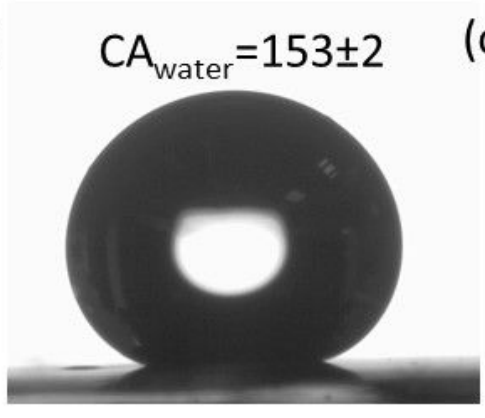

(d)

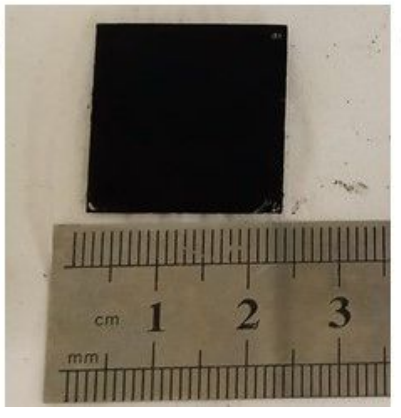

(e)

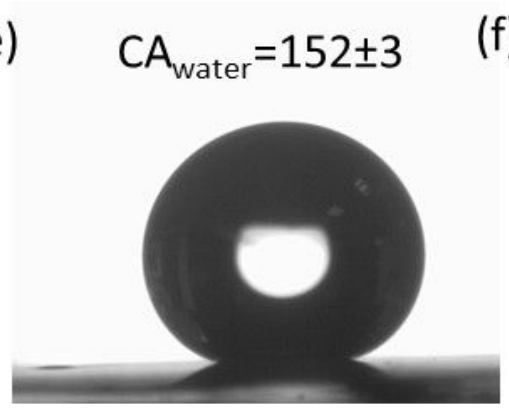

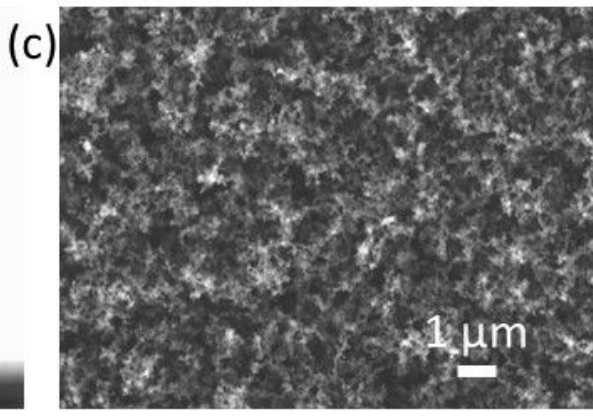

(f)

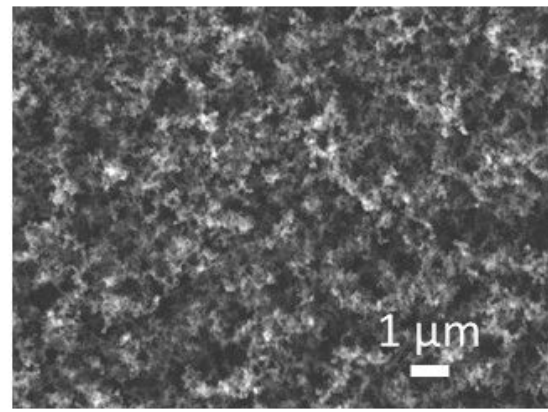

Figure S9. Mechanical stability of the candle soot surface. Before exposure to the water stream at the velocity of $0.32 \mathrm{~m} / \mathrm{s}$ : a) optical image b) water contact angle c) SEM image. After exposure: d) optical image e) water contact angle f) SEM image.

Table S1. Leaching tests of the CSNPs coated surfaces. Total organic carbon (TOC) and surface contact angle were measured after applied conditions.

\begin{tabular}{|c|c|c|c|}
\hline Conditions & $\begin{array}{c}\text { PDMS spacer } \\
\text { TOC }\left(\mathrm{mg} \mathrm{L}^{-1}\right)\end{array}$ & $\begin{array}{c}\text { CSNPs spacer } \\
\text { TOC }\left(\mathrm{mg} \mathrm{L}^{-1}\right)\end{array}$ & $\begin{array}{c}\text { CSNPs spacer } \\
\left.\text { Contact angle }{ }^{\circ}\right)\end{array}$ \\
\hline 1 min sonication & 1.46 & 1.19 & $154.2 \pm 0.5$ \\
\hline 2 min sonication & 1.65 & 1.71 & $153.4 \pm 0.4$ \\
\hline 5 min sonication & 1.61 & 1.63 & $154.8 \pm 0.3$ \\
\hline $\mathrm{pH} 2,5$ min sonic. & 1.81 & 1.21 & $155.6 \pm 0.4$ \\
\hline $\mathrm{pH} 6.5,5$ min sonic. & 1.52 & 1.75 & $155.8 \pm 0.5$ \\
\hline $\mathrm{pH} 10,5 \mathrm{~min}$ sonic. & 1.41 & 2.14 & $154.9 \pm 0.4$ \\
\hline $40^{\circ} \mathrm{C}, 5 \mathrm{~min}$ & 1.58 & 1.04 & $155.2 \pm 0.7$ \\
\hline $60^{\circ} \mathrm{C}, 5 \mathrm{~min}$ & 1.69 & 0.91 & $155.8 \pm 0.2$ \\
\hline $80^{\circ} \mathrm{C}, 5 \mathrm{~min}^{-1}$ & 1.61 & 0.97 & $155.7 \pm 0.6$ \\
\hline $50 \mathrm{~mL} \mathrm{~min}^{-1}$ & 0.47 & 0.29 & $155.8 \pm 0.5$ \\
\hline $100 \mathrm{~mL} \mathrm{~min}^{-1}$ & 0.61 & 0.44 & $155.2 \pm 0.4$ \\
\hline $200 \mathrm{~mL} \mathrm{~min}^{-1}$ & 0.43 & 0.37 & $155.3 \pm 0.7$ \\
\hline
\end{tabular}


